\title{
The Chinese Medicinal Formulation Guzhi Zengsheng Zhitongwan Modulates Chondrocyte Structure, Dynamics, and Metabolism by Controlling Multiple Functional Proteins
}

\author{
Baojin Yao, ${ }^{1}$ Bocheng Lu, ${ }^{2}$ Mei Zhang, ${ }^{3}$ Hongwei Gao, ${ }^{2}$ \\ Xiangyang Leng $\mathbb{D}{ }^{2}$, and Daqing Zhao $\mathbb{D}^{1}$ \\ ${ }^{1}$ Jilin Ginseng Academy, Changchun University of Chinese Medicine, Changchun, Jilin 130117, China \\ ${ }^{2}$ The Affiliated Hospital of Changchun University of Chinese Medicine, Changchun, Jilin 130117, China \\ ${ }^{3}$ Innovation Practice Center, Changchun University of Chinese Medicine, Changchun, Jilin 130117, China \\ Correspondence should be addressed to Xiangyang Leng; leng_xiangyang@163.com and Daqing Zhao; zhaodaqing1963@163.com
}

Received 25 September 2018; Accepted 8 November 2018; Published 22 November 2018

Academic Editor: Kibret Mequanint

Copyright (C) 2018 Baojin Yao et al. This is an open access article distributed under the Creative Commons Attribution License, which permits unrestricted use, distribution, and reproduction in any medium, provided the original work is properly cited.

Traditional Chinese medicine is one of the oldest medical systems in the world and has its unique principles and theories in the prevention and treatment of human diseases, which are achieved through the interactions of different types of materia medica in the form of Chinese medicinal formulations. GZZSZTW, a classical and effective Chinese medicinal formulation, was designed and created by professor Bailing Liu who is the only national medical master professor in the clinical research field of traditional Chinese medicine and skeletal diseases. GZZSZTW has been widely used in clinical settings for several decades for the treatment of joint diseases. However, the underlying molecular mechanisms are still largely unknown. In the present study, we performed quantitative proteomic analysis to investigate the effects of GZZSZTW on mouse primary chondrocytes using state-of-the-art iTRAQ technology. We demonstrated that the Chinese medicinal formulation GZZSZTW modulates chondrocyte structure, dynamics, and metabolism by controlling multiple functional proteins that are involved in the cellular processes of DNA replication and transcription, protein synthesis and degradation, cytoskeleton dynamics, and signal transduction. Thus, this study has expanded the current knowledge of the molecular mechanism of GZZSZTW treatment on chondrocytes. It has also shed new light on possible strategies to further prevent and treat cartilage-related diseases using traditional Chinese medicinal formulations.

\section{Introduction}

Chinese materia medica (CMM), the main form of traditional Chinese medicine (TCM), is widely used by a large, global population. The World Health Organization (WHO) also encourages member countries to include traditional medicine and materia medica in their primary healthcare systems. Therefore, TCM and CMM have been used by people and communities all over the world [1]. TCM is one of the oldest medical systems in the world and has unique principles and theories in the prevention and treatment of human diseases. The effects of TCM are achieved through the interactions of different types of materia medica, which are combined to make Chinese medicinal formulations. These formulations have been used and tested in humans for many centuries, from ancient times to today. Furthermore, tens of thousands of clinical reports have been published up till now [2].

Osteoarthritis (OA) is the most common joint disease characterized by articular cartilage degeneration accompanied by inflammation and osteophyte formation. There are some remedies for OA to relieve pain, such as analgesics, nonsteroidal anti-inflammatory drugs (NSAIDs), or joint replacement surgery. However, none of these treatments are sufficient to prevent the progression of OA. This is due to the poor repair and regeneration potentials of chondrocytes, the only cell type in cartilage, which controls cartilage structure and function [3]. Moreover, $\mathrm{OA}$ is a leading cause of disability worldwide, and, currently, there is no effective treatment for OA patients [4]. 
Guzhi Zengsheng Zhitongwan (GZZSZTW) is an effective Chinese medicinal formulation and has been used for the treatment of $\mathrm{OA}$ patients in the Affiliated Hospital of Changchun University of Chinese Medicine for several decades. This formula is orally administered in the form of pills prepared in boiled water, which are only used in the hospital's internal preparation center to treat osteoarthritis patients. However, our knowledge of the regulatory role of GZZSZTW on primary chondrocytes and the underlying mechanism remains largely unknown. In the present study, we performed quantitative proteomic analysis to investigate the effects of GZZSZTW on mouse primary chondrocytes using state-of-the-art iTRAQ technology. We demonstrated that the Chinese medicinal formulation GZZSZTW modulates chondrocyte structure, dynamics, and metabolism by controlling multiple functional proteins that were involved in the cellular processes of DNA replication and transcription, protein synthesis and degradation, cytoskeleton dynamics, and signal transduction.

\section{Materials and Methods}

2.1. Preparation of the GZZSZTW Aqueous Extract. GZZSZTW medicinal formulation, which consisted of Rehmannia glutinosa (Gaertn.) DC., Spatholobus suberectus Dunn, Epimedium brevicornu Maxim (K.S.Hao), Raphanus sativus L. (Hook. f. \& T. Anderson) (baked), Drynaria fortunei (Kunze ex Mett.) J.Sm. (baked), Cynomorium coccineum subsp. songaricum (Rupr.) (J.Léonard), and Cibotium barometz (L.) (J.Sm), was provided by the Affiliated Hospital of Changchun University of Chinese Medicine (Changchun, China). The mixture of GZZSZTW was immersed in distilled water for $30 \mathrm{~min}$. Subsequently, the mixture was decocted with a 10 -fold volume of distilled water by a refluxing method and was then filtered through a $0.45 \mu \mathrm{m}$ Hollow Fiber Cartridge (GE Healthcare, USA). The filtrate was freeze-dried using a Heto PowerDry LL3000 Freeze Dryer (Thermo, USA) and stored at $-80^{\circ} \mathrm{C}$ [5].

2.2. Primary Chondrocytes Isolation and Treatment. Animal experiments were approved by the Ethical Committee for Animal Research of Changchun University of Chinese Medicine. Primary chondrocytes were isolated and cultured as previously described [6]. Briefly, rib cages of C57BL/6J neonatal mice were dissected under sterile conditions and the cartilage was carefully separated and digested with $3 \mathrm{mg} / \mathrm{ml}$ collagenase D for $45 \mathrm{~min}$ and then overnight with $0.5 \mathrm{mg} / \mathrm{ml}$ collagenase D (Sigma, USA). The cells released from the tissue were centrifuged and suspended in DMEM/F12 medium (Thermo, USA) containing 5\% FCS (Thermo, USA) and $1 \%$ penicillin/streptomycin (Sigma, USA). Primary chondrocytes were seeded into 6 -well culture dishes at a density of $1 \times 10^{5}$ cells/well $(2 \mathrm{ml}$ per well $)$ and cultured at $37^{\circ} \mathrm{C}$ in an incubator (Thermo, USA) containing $5 \% \mathrm{CO}_{2}$ for $4 \mathrm{~h}$. Then, the culture medium was discarded, and each well was completely rinsed with fresh culture medium. The chondrocytes were treated with either $0.8 \mathrm{mg} / \mathrm{ml}$ GZZSZTW dissolved in culture medium or plain culture medium for $24 \mathrm{~h}$ [5].
2.3. Preparation of Protein Extracts. The culture medium was discarded, and each well was completely rinsed with ice-cold phosphate-buffered saline $(\mathrm{pH} 7.4,137 \mathrm{mM} \mathrm{NaCl}, 2.7 \mathrm{mM}$ $\mathrm{KCl}, 4.3 \mathrm{mM} \mathrm{Na} \mathrm{HPO}_{4}$, and $\left.1.4 \mathrm{mM} \mathrm{K \textrm {K } _ { 2 }} \mathrm{PO}_{4}\right)$. Primary chondrocytes were suspended in the lysis buffer (7 M Urea, $2 \mathrm{M}$ Thiourea, 4\% CHAPS, $40 \mathrm{mM}$ Tris- $\mathrm{HCl}$ (pH 8.5), $1 \mathrm{mM}$ PMSF, and $2 \mathrm{mM}$ EDTA) and sonicated for $30 \mathrm{~min}$ on ice. Then, $10 \mathrm{mM}$ DTT (Sigma, USA) was added and incubated at $56^{\circ} \mathrm{C}$ for $1 \mathrm{~h}$ followed by the addition of $55 \mathrm{mM}$ IAM (Sigma, USA) and incubation in the darkroom at room temperature for $45 \mathrm{~min}$. The proteins were precipitated with ice-cold acetone $(1: 5, \mathrm{v} / \mathrm{v})$ at $-20^{\circ} \mathrm{C}$ for $2 \mathrm{~h}$. After centrifugation at $4^{\circ} \mathrm{C}$ for $15 \mathrm{~min}$, the pellet was dissolved in $0.5 \mathrm{M}$ TEAB (Sigma, USA) and sonicated for $20 \mathrm{~min}$ on ice. The supernatant was obtained by centrifuging again and protein concentration was determined using a Bradford protein assay kit (Bio-Rad, USA) [7].

\subsection{Peptide Labeling and Strong Cation Exchange Fraction-} ation. A total of $100 \mu \mathrm{g}$ of protein per sample was used for iTRAQ labeling. Protein samples were digested with trypsin (Sigma, USA) with a mass ratio of $20: 1$ at $37^{\circ} \mathrm{C}$ for $12 \mathrm{~h}$. The digested proteins were freeze-dried, reconstituted in 0.5 M TEAB (Sigma, USA), and labeled using the iTRAQ Reagent 8 Plex One Assay Kit (AB Sciex, USA) according to the manufacturer's instructions [8]. The untreated blank group was labeled with iTRAQ tag 114 and the GZZSZTW group was labeled with tag 115 . The labeled peptide mixtures from the two groups were then equally mixed and dried using a speed-vacuum centrifuge (Thermo, USA). The dried labeled peptides were dissolved in $4 \mathrm{~mL}$ of strong cation exchange (SCX) buffer $\left(25 \mathrm{mM} \mathrm{NaH}{ }_{2} \mathrm{PO}_{4}\right.$ in $25 \%$ acetonitrile, $\mathrm{pH}$ 2.7) and fractionated using an Ultremex strong cation exchange column (Phenomenex, USA) on the LC$20 \mathrm{AB}$ high-performance liquid chromatography platform (Shimadzu, Japan) [9].

\subsection{High-Performance Liquid Chromatography and Mass} Spectrometry Analysis. The fractions were further identified and quantified by ultra-high-performance liquid chromatography using a nanoACQuity (Waters, USA) coupled with a Triple TOF 5600 tandem mass spectrometer (AB SCIEX, USA) and subsequently analyzed according to previously described methods [10]. Briefly, online trapping and desalting were performed using microfluidic traps and nanofluidic columns packed with Symmetry C18 (Waters, USA) and analytical separation was carried out using nanofluidic columns packed with BEH130 C18 (Waters, USA). Data were acquired on a Triple TOF 5600 platform using an ion spray voltage of $2.5 \mathrm{kV}$, a curtain gas of $30 \mathrm{psi}$, a nebulizer gas of $15 \mathrm{psi}$, and an interface heater temperature of $150^{\circ} \mathrm{C}$. The MS was operated with a resolving power of greater than or equal to 30,000 FWHM for the TOF-MS scans. For information-dependent acquisition, the survey scans were acquired at $250 \mathrm{~ms}$, and as many as 30 product ion scans were collected when exceeding a threshold of 120 counts per second and with a $2+$ to $5+$ charge-state. The Q2 transmission window was set to $100 \mathrm{Da}$ for $100 \%$. A sweeping collision energy setting of $35 \pm 5 \mathrm{eV}$ 


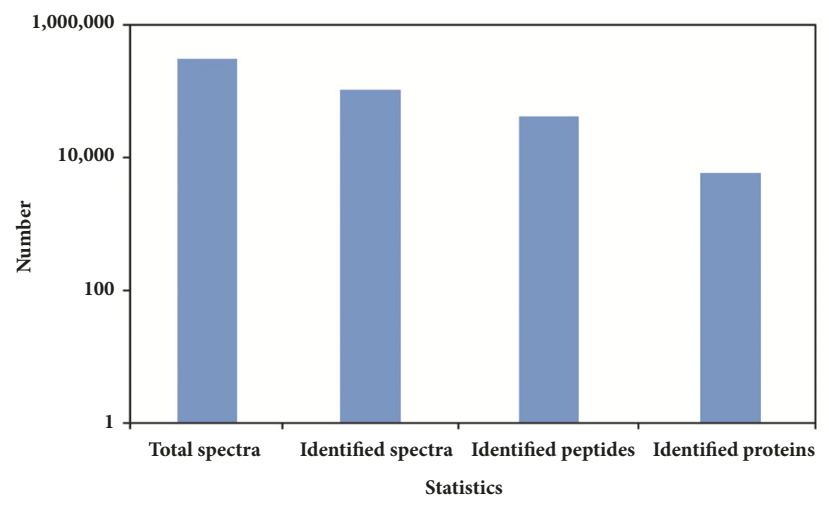

(a)

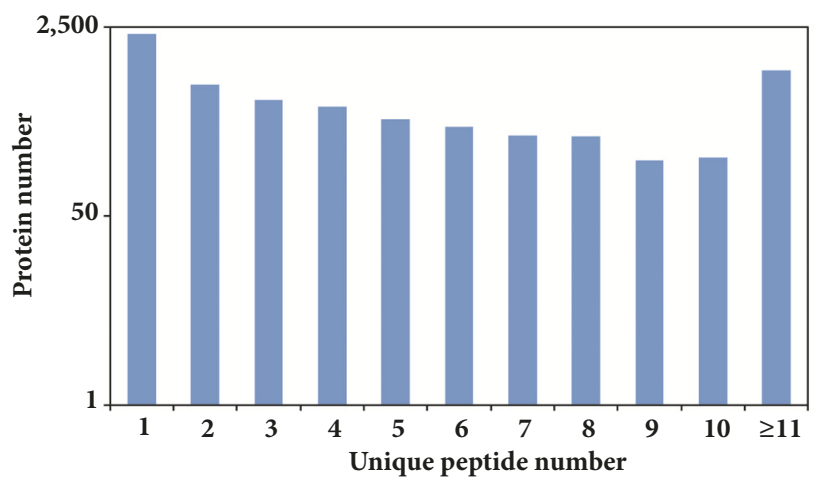

(c)

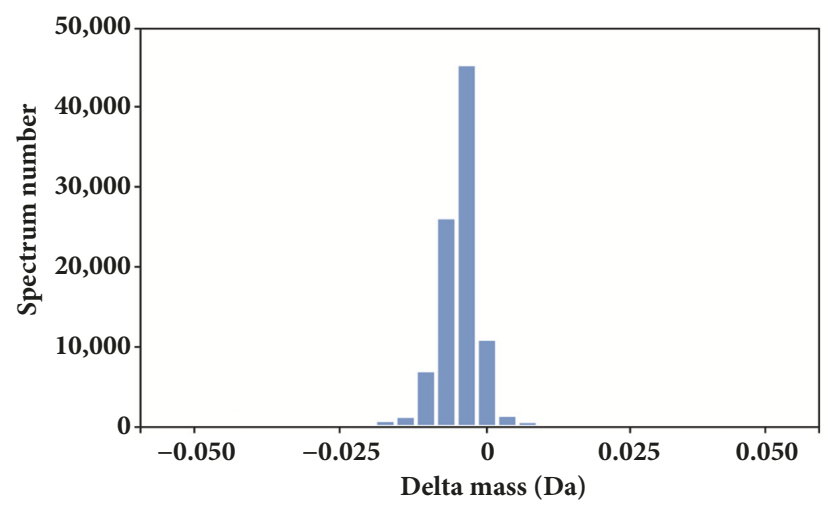

(b)

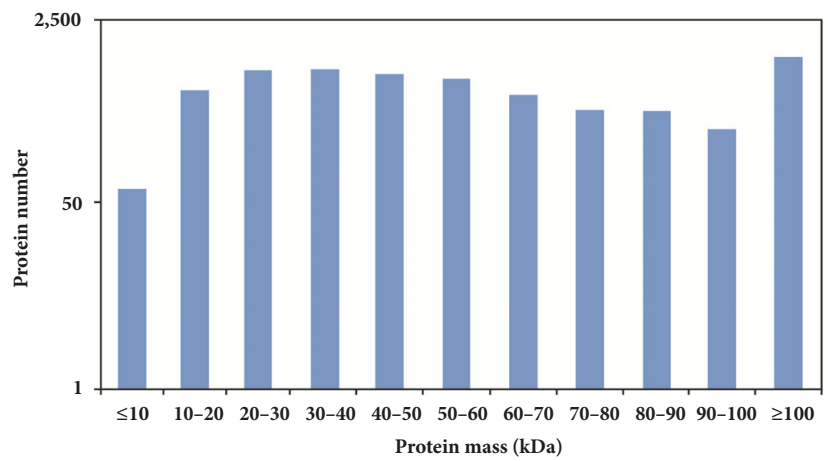

(d)

FIGURE 1: Statistics of protein identification. (a) Numbers of spectra, peptides, and proteins that were identified by iTRAQ identification. (b) The relationship between spectra number and delta mass. (c) Number of unique peptides that match associated proteins. (d) Molecular weight distribution of the proteins that were identified from the iTRAQ analysis.

coupled with the iTRAQ adjusted rolling collision energy was applied to all the precursor ions for collision-induced dissociation. The dynamic exclusion was set for $1 / 2$ of the peak width, and then, the precursor was refreshed off the exclusion list.

2.6. Data Analysis. The collected raw data were processed using the Proteome Discoverer software (Thermo, USA). The Mascot search engine (Matrix Sciences, UK) was used to search against the NCBI nonredundant protein (https://www.ncbi .nlm.nih.gov/refseq/) and UniProt databases (http://www .uniprot.org/) [11]. The search results were integrated by the IPeak program [12]. The protein quantification was performed using the IQuant software [13]. The following criteria were used for screening differentially expressed proteins: fold change $\geq 1.5$ or $\leq 0.67$ and $p$ value $\leq 0.05$ [14]. Functional annotations were further conducted by searching these proteins against the KEGG (http://www.genome.jp/ kegg/pathway.html), eggNOG (http://eggnogdb.embl.de/), and WoLF PSORT databases (https://wolfpsort.hgc.jp/) [15].

2.7. Parallel Reaction Monitoring Validation. The parallel reaction monitoring (PRM) assays were used to further validate the expression levels of differentially expressed proteins as previously described [16]. Briefly, SpectroDive software (version 8.0, Biognosys AG, Zurich, Switzerland) was used to generate the spectral library, and the target candidates were selected from the Data Dependent Acquisition (DDA) proteomic runs that were acquired using a Q-EXACTIVE instrument. Data analysis was performed using default settings with minor modifications. Protein fold changes were estimated using Student's $t$-test and the cutoff was 0.05 .

\section{Results}

3.1. Protein Identification. A total of 312,285 spectra were detected by iTRAQ and 106,325 were identified. Additionally, 41,555 peptides matched to these spectra were identified. In total, based on these identified peptides, 6,026 proteins were identified as shown in Figure 1(a). The newly identified peptides were detected with a delta mass between -0.025 and $+0.025 \mathrm{Da}$ (Figure 1(b)). A majority of the identified proteins consisted of at least two unique peptides (Figure 1(c)). The protein masses of more than half of these proteins ranged from 10 to $70 \mathrm{kDa}$, though their molecular weights covered a wide range (Figure $1(\mathrm{~d})$ ).

3.2. Functional Annotation of the Differentially Expressed Proteins. We identified a total of 84 differentially expressed proteins according to the iTRAQ analysis. Among these proteins, the expression levels of 67 proteins were significantly increased and 17 proteins were significantly decreased after 
TABLE 1: KEGG enrichment analysis of differentially expressed proteins (GZZSZTW versus Blank).

\begin{tabular}{ll}
\hline Pathways & Number of genes \\
\hline Translation & 12 \\
Transport and catabolism & 6 \\
Signal transduction & 6 \\
Immune system & 5 \\
Infectious diseases: viral & 4 \\
Cell growth and death & 3 \\
Cancers: overview & 3 \\
Endocrine system & 3 \\
Cellular community - eukaryotes & 2 \\
Transcription & 2 \\
Cardiovascular diseases & 2 \\
Infectious diseases: bacterial & 2 \\
Neurodegenerative diseases & 2 \\
Carbohydrate metabolism & 2 \\
Global and overview maps & 2 \\
Digestive system & 2 \\
Cell motility & 1 \\
Membrane transport & 1 \\
Signaling molecules and interaction & 1 \\
Drug resistance: antineoplastic & 1 \\
Immune diseases & 1 \\
Infectious diseases: parasitic & 1 \\
Substance dependence & 1 \\
Excretory system & 1 \\
Sensory system & \\
\hline
\end{tabular}

TABLE 2: Subcellular distribution of differentially expressed proteins (GZZSZTW versus Blank).

\begin{tabular}{lc}
\hline Subcellular location & Number of genes \\
\hline Nucleus & 35 \\
Cytoplasm & 18 \\
Extracellular space & 14 \\
Mitochondria & 6 \\
Plasma membrane & 6 \\
Cytoplasm \& nucleus & 3 \\
Cytoskeleton & 2 \\
\hline
\end{tabular}

GZZSZTW treatment. We first performed KEGG pathway enrichment analysis. These differentially expressed proteins were mapped to 25 KEGG pathways, as shown in Table 1. The top eight enriched pathways, according to the numbers of mapped proteins, included translation (12), transport and catabolism (6), signal transduction (6), immune system (5), infectious diseases (specifically viral) (4), cell growth and death (3), cancers (overview) (3), and endocrine system (3).

We then analyzed the subcellular localization of these differentially expressed proteins. As shown in Table 2, the majority of these differentially expressed proteins were distributed in the nucleus (35), cytoplasm (18), and extracellular space (14).
We further analyzed the functions of these differentially expressed proteins based on eggNOG classification. As shown in Table 3, the majority of these differentially expressed proteins were classified into the following functional categories: cytoskeleton (17), posttranslational modification/protein turnover/chaperones (14), signal transduction mechanisms (11), chromatin structure and dynamics (11), translation/ribosomal structure and biogenesis (10), transcription (8), extracellular structures (7), or intracellular trafficking/secretion and vesicular transport (5). To fully explore the mechanisms by which GZZSZTW regulates chondrocyte protein expression, we further dissected the protein expression patterns based on the eggNOG classification in detail.

The 17 differentially expressed proteins that were classified under the category of cytoskeleton, as shown in Table 4, were significantly increased after GZZSZTW treatment; among these were several subtypes of keratin types I and II (Krt79, Krt17, Krt15, Krt42, Krt16, Krt10, Krt5, Krt6a, Krt14, Krt1, and Krt2), tubulin alpha-1B chain (Tubalb), MARCKS-related protein (Marcksl1), desmoplakin (Dsp), septin-7 (Sept7), tropomodulin-2 (Tmod2), and gammasynuclein (Sncg).

Among the 14 differentially expressed proteins from the category of posttranslational modification, protein turnover, and chaperones, as shown in Table 5, the levels of 8 proteins were significantly increased after GZZSZTW treatment: pregnancy zone protein (Pzp), MARCKS-related protein (Marcksl1), ovostatin homolog (Ovos), tuberoinfundibular peptide of 39 residues (Pth2), calpastatin (Cast), UBX domain-containing protein 4 (Ubxn4), matrix Gla protein (Mgp), and gamma-synuclein (Sncg). The levels of 6 proteins were significantly decreased under GZZSZTW treatment: alpha-fetoprotein (Afp), lactotransferrin (Ltf), serum albumin (Alb), chymotrypsinogen B (Ctrb1), alpha-2-HSglycoprotein (Ahsg), and kallikrein 1-related peptidase b1 (Klk1b1).

A majority of the 11 differentially expressed proteins from the category of signal transduction mechanisms, as shown in Table 6, were significantly increased after GZZSZTW treatment, including granulocyte colony-stimulating factor receptor (Csf3r), death-associated protein 1 (Dap), keratin type II cytoskeletal 79 (Krt79), MARCKS-related protein (Marcksl1), $\mathrm{PH}$ domain leucine-rich repeat-containing protein phosphatase 2 (Phlpp2), endophilin-B1 (Sh3glb1), tuberoinfundibular peptide of 39 residues (Pth2), craniofacial development protein 1 (Cfdp1), a-kinase anchor protein 8 (Akap8), and disabled homolog 2 (Dab2).

A majority of the 11 differentially expressed proteins from the category of chromatin structure and dynamics, as shown in Table 7, were significantly increased after GZZSZTW treatment; among them were parathymosin (Ptms), histone H1.5 (Histlhlb), methyl-CpG-binding protein 2 (Mecp2), high mobility group protein HMG-I/HMG-Y (Hmgal), histone H1.3 (Histlhld), histone H1.4 (Histlhle), glyceraldehyde-3phosphate dehydrogenase (Gapdh), histone H1.1 (Histlhla), histone H2A.V (H2afv), and a-kinase anchor protein 8 (Akap8).

A majority of the 10 differentially expressed proteins that were classified under the category of translation, ribosomal 
TABLE 3: Statistics of eggNOG classification of differentially expressed proteins (GZZSZTW versus Blank).

\begin{tabular}{lc}
\hline Categories & Number of genes \\
\hline Cytoskeleton & 17 \\
Posttranslational modification, protein turnover, chaperones & 14 \\
Signal transduction mechanisms & 11 \\
Chromatin structure and dynamics & 11 \\
Translation, ribosomal structure and biogenesis & 10 \\
Transcription & 8 \\
Extracellular structures & 7 \\
Intracellular trafficking, secretion, and vesicular transport & 5 \\
RNA processing and modification & 2 \\
Amino acid transport and metabolism & 2 \\
Inorganic ion transport and metabolism & 2 \\
Carbohydrate transport and metabolism & 1 \\
Replication, recombination and repair & 1 \\
Cell cycle control, cell division, chromosome partitioning & 1 \\
Energy production and conversion & 1 \\
\hline
\end{tabular}

TABLE 4: Differentially expressed proteins that were classified into cytoskeleton (GZZSZTW versus Blank).

\begin{tabular}{lcc}
\hline Protein name & Fold change & p value \\
\hline Keratin, type II cytoskeletal 79 (Krt79) & 2.660 & 0.001 \\
Keratin, type I cytoskeletal 17 (Krt17) & 2.446 & 0.044 \\
Tubulin alpha-1B chain (Tuba1b) & 2.309 & 0.015 \\
Keratin, type I cytoskeletal 15 (Krt15) & 2.187 & 0.022 \\
Keratin, type I cytoskeletal 42 (Krt42) & 2.112 \\
Keratin, type I cytoskeletal 16 (Krt16) & 2.027 \\
Keratin, type I cytoskeletal 10 (Krt10) & 2.023 \\
MARCKS-related protein (Marcksl1) & 1.993 \\
Keratin, type II cytoskeletal 5 (Krt5) & 1.973 \\
Keratin, type II cytoskeletal 6A (Krt6a) & 1.908 \\
Keratin, type I cytoskeletal 14 (Krt14) & 1.727 \\
Keratin, type II cytoskeletal 1 (Krt1) & 1.708 \\
Desmoplakin (Dsp) & 1.661 \\
Septin-7 (Sept7) & 1.623 \\
Keratin, type II cytoskeletal 2 (Krt2) & 1.575 \\
Tropomodulin-2 (Tmod2) & 1.558 \\
Gamma-synuclein (Sncg) & 1.521 \\
\hline
\end{tabular}

structure, and biogenesis were significantly increased after GZZSZTW treatment, as shown in Table 8; among them were subtypes of $40 \mathrm{~S}$ and $60 \mathrm{~S}$ ribosomal proteins (Rps19, Rps27l, Rpl34, Rpl37, Rps28, Rps23, Rpl35, and Rpl36) and Eukaryotic translation initiation factor $1 \mathrm{~A}, \mathrm{X}$-chromosomal (Eiflax).

The details of the differentially expressed proteins in the categories of transcription, extracellular structures and intracellular trafficking/secretion/vesicular transport, RNA processing and modification, amino acid transport and metabolism, inorganic ion transport and metabolism, carbohydrate transport and metabolism, replication/recombination and repair, cell cycle control/cell division/chromosome partitioning, and energy production/conversion are described in Table 9.
3.3. Parallel Reaction Monitoring Validation. Differentially expressed proteins identified by iTRAQ were further validated by a parallel reaction monitoring (PRM) assay. Twelve differentially expressed proteins, Krt42, Krt5, Krt16, Mgp, Pzp, Rpl35, Rps23, Cfdp1, Ptms, Histlhlb, Mecp2, and Histlhla, were randomly selected for PRM analysis. As shown in Table 10, the fold changes were in agreement with the findings of the iTRAQ analysis with a $p$ value $\leq 0.05$.

\section{Discussion}

Currently, there is still no effective western medication for OA. The medications that are used clinically can only help to 
TABLE 5: Differentially expressed proteins that were classified into posttranslational modification, protein turnover, chaperones (GZZSZTW versus Blank).

\begin{tabular}{lcc}
\hline Protein name & Fold change & Q value \\
\hline Pregnancy zone protein (Pzp) & 2.088 & 0.001 \\
MARCKS-related protein (Marcksl1) & 1.993 & 0.012 \\
Ovostatin homolog (Ovos) & 1.955 & 0.007 \\
Tuberoinfundibular peptide of 39 residues (Pth2) & 1.688 & 0.003 \\
Calpastatin (Cast) & 1.631 & 0.001 \\
UBX domain-containing protein 4 (Ubxn4) & 1.580 & 0.001 \\
Matrix Gla protein (Mgp) & 1.535 & 0.003 \\
Gamma-synuclein (Sncg) & 1.521 & 0.011 \\
Alpha-fetoprotein (Afp) & 0.637 & 0.001 \\
Lactotransferrin (Ltf) & 0.550 & 0.003 \\
Serum albumin (Alb) & 0.545 \\
Chymotrypsinogen B (Ctrb1) & 0.511 \\
Alpha-2-HS-glycoprotein (Ahsg) & 0.364 & 0.001 \\
Kallikrein 1-related peptidase b1 (Klk1b1) & 0.291 \\
\hline
\end{tabular}

TABLE 6: Differentially expressed proteins that were classified into signal transduction mechanisms (GZZSZTW versus Blank).

\begin{tabular}{lcc}
\hline Protein name & Fold change & Q value \\
\hline Granulocyte colony-stimulating factor receptor (Csf3r) & 8.552 & 0.001 \\
Death-associated protein 1 (Dap) & 3.140 & 0.001 \\
Keratin, type II cytoskeletal 79 (Krt79) & 2.660 & 0.001 \\
MARCKS-related protein (Marcksl1) & 1.993 & 0.012 \\
PH domain leucine-rich repeat-containing protein phosphatase 2(Phlpp2) & 1.935 & 0.003 \\
Endophilin-B1 (Sh3glb1) & 1.724 \\
Tuberoinfundibular peptide of 39 residues (Pth2) & 1.688 \\
Craniofacial development protein 1 (Cfdp1) & 1.683 \\
A-kinase anchor protein 8(Akap8) & 1.508 \\
Disabled homolog 2 (Dab2) & 1.500 \\
Alpha-2-HS-glycoprotein (Ahsg) & 0.364 \\
\hline
\end{tabular}

relieve pain and have adverse side effects including cardiovascular, renal, and gastrointestinal complications; hepatotoxicity and cognitive impairment; and injuries [17]. Recently, Chen and colleagues conducted a meta-analysis and demonstrated that traditional Chinese medicinal formulations were safer and more effective for reducing pain and improving overall physical performance and wellness in treatments of knee OA, and they had a lower risk of adverse events than standard western medication treatments [18]. Therefore, it is time for us to consider using traditional Chinese medications for the treatment of OA, since most of the TCM formulations have been used for many centuries and have been investigated in long-term clinical trials in human beings. The most important mission for us is to elucidate their underlying mechanisms in the prevention and treatment of human diseases so that TCM will be accepted and used all over the world.

GZZSZTW, a classical and effective formulation, is designed and created by professor Bailing Liu who is the only national medical master professor in the clinical research field of TCM and skeletal diseases. For several decades, it has been widely used in the Affiliated Hospital of Changchun University of Chinese Medicine to treat joint diseases. However, the underlying molecular mechanisms are still largely unknown. In the present study, we performed a quantitative proteomic analysis to investigate the effects of GZZSZTW on mouse primary chondrocytes using state-of-the-art iTRAQ technology. We aimed to demonstrate the direct effects of GZZSZTW on chondrocytes and further dissect the regulating factors and related pathways.

We first analyzed the differentially expressed proteins that mapped to KEGG pathways. We demonstrated that GZZSZTW treatment significantly changed the expression levels of multiple proteins involved in $25 \mathrm{KEGG}$ pathways that play important roles in regulating multiple biological processes in chondrocytes, such as cellular processes, environmental information processing, genetic information processing, human diseases, metabolism, and organismal systems. The pathways of cell growth and death, cell motility, cellular community-eukaryotes, transport, and catabolism were classified into cellular processes. The pathways of membrane transport, signal transduction, signaling molecules, and interaction were classified into environmental information processing. The pathways of transcription and translation were classified into genetic information processing. 
TABLE 7: Differentially expressed proteins that were classified into chromatin structure and dynamics (GZZSZTW versus Blank).

\begin{tabular}{lcc}
\hline Protein name & Fold change & Q value \\
\hline Parathymosin (Ptms) & 3.085 & 0.001 \\
Histone H1.5 (Histlhlb) & 2.104 & 0.001 \\
Methyl-CpG-binding protein 2 (Mecp2) & 2.056 & 0.040 \\
High mobility group protein HMG-I/HMG-Y (Hmgal) & 1.959 & 0.001 \\
Histone H1.3 (Histlhld) & 1.950 & 0.005 \\
Histone H1.4 (Histlhle) & 1.901 & 0.001 \\
Glyceraldehyde-3-phosphate dehydrogenase (Gapdh) & 1.547 & 0.001 \\
Histone H1.1 (Histlhla) & 1.530 & 0.001 \\
Histone H2A.V (H2afv) & 1.515 \\
A-kinase anchor protein 8 (Akap8) & 1.508 & 0.007 \\
Tudor domain-containing protein 1 (Tdrd1) & 0.619 & 0.008 \\
\hline
\end{tabular}

TABLE 8: Differentially expressed proteins that were classified into translation, ribosomal structure, and biogenesis (GZZSZTW versus Blank).

\begin{tabular}{lcc}
\hline Protein name & Fold change & Q value \\
\hline 40S ribosomal protein S19 (Rps19) & 1.843 & 0.001 \\
40S ribosomal protein S27-like (Rps27l) & 1.713 & 0.026 \\
60S ribosomal protein L34 (Rpl34) & 1.645 & 0.001 \\
60S ribosomal protein L37 (Rpl37) & 1.582 & 0.008 \\
40S ribosomal protein S28 (Rps28) & 1.580 & 0.001 \\
40S ribosomal protein S23 (Rps23) & 1.546 & 0.001 \\
60S ribosomal protein L35 (Rpl35) & 1.537 & 0.001 \\
Eukaryotic translation initiation factor 1A, X-chromosomal (Eiflax) & 1.512 & 0.001 \\
60S ribosomal protein L36 (Rpl36) & 1.508 & 0.037 \\
60S acidic ribosomal protein P1 (Rplp1) & 0.332 & 0.001 \\
\hline
\end{tabular}

The pathways of cancers, cardiovascular diseases, drug resistance, immune diseases, infectious diseases, neurodegenerative diseases, and substance dependence were classified into the regulation of human diseases. The pathways of carbohydrate metabolism and global and overview maps were classified into metabolism processes. The pathways of digestive, endocrine, excretory, immune, and sensory systems were classified into the regulation of organismal systems. These results suggest that GZZSZTW treatment significantly affects protein synthesis and changes the expression levels of functional proteins that participate in chondrocyte growth, metabolism, signaling transduction, and the regulation of disease.

Consistent with the results discussed above, the majority of the differentially expressed proteins were localized in the nucleus, cytoplasm, and extracellular space, which are the crucial places for gene transcription and regulation, signaling transduction and extracellular matrix secretion, and maintenance in chondrocytes. Unlike other tissues, cartilage is a resilient, smooth, and highly specialized connective tissue. Chondrocytes are the only type of cell in cartilage and they produce a large amount of extracellular matrices to regulate chondrocyte metabolism and function [19].

These differentially expressed proteins were further annotated based on BLAST searches against the eggNOG database that provides orthologous groups of proteins at different taxonomic levels with integrated and summarized functional annotations [20]. Our results demonstrated that the majority of these differentially expressed proteins were classified into the categories of cytoskeleton, posttranslational modification/protein turnover/chaperones, signal transduction mechanisms, chromatin structure and dynamics/translation, and ribosomal structure and biogenesis. These results were consistent with our previous KEGG pathway and subcellular localization analysis. Therefore, we further analyzed these differentially expressed proteins in detail according to the eggNOG annotation.

According to the eggNOG annotation, there were 17 differentially expressed proteins that were from the cytoskeleton category. Their expression levels in primary chondrocytes were significantly increased after GZZSZTW treatment. These proteins mainly consisted of type I and type II keratins including Krt79, Krt17, Krt15, Krt42, Krt16, Krt10, Krt5, Krt6a, Krt14, Krt1, and Krt2. Keratins are intermediate filamentforming proteins that provide mechanical support for the cytoskeleton of many cell types, such as chondrocytes. Type I and type II keratins play crucial roles in diverse cellular processes including cell shape adaptation, division, migration and extracellular matrix assembly, and secretion [21, 22]. Our results showed that the expression levels of several other cytoskeleton proteins including Tubalb, Marcksl1, Dsp, Sept7, Tmod2, and Sncg were also increased after GZZSZTW treatment. Tubalb is one of the major components of microtubules, which are one of the three principal types of protein 
TABLE 9: Differentially expressed proteins that were classified into other eggNOG categories (GZZSZTW versus Blank).

\begin{tabular}{|c|c|c|}
\hline Protein name & Fold change & Q value \\
\hline \multicolumn{3}{|l|}{ Transcription } \\
\hline Death-associated protein 1 (Dap) & 3.140 & 0.001 \\
\hline Protein S100-A1 (S100a1) & 2.538 & 0.009 \\
\hline Methyl-CpG-binding protein 2 (Mecp2) & 2.056 & 0.040 \\
\hline High mobility group protein HMG-I/HMG-Y (Hmgal) & 1.959 & 0.001 \\
\hline Prothymosin alpha (Ptma) & 1.890 & 0.016 \\
\hline Nuclear factor 1 A-type (Nfia) & 1.787 & 0.028 \\
\hline Tudor domain-containing protein 1 (Tdrd1) & 0.619 & 0.001 \\
\hline Lactotransferrin (Ltf) & 0.550 & 0.003 \\
\hline \multicolumn{3}{|l|}{ Extracellular structures } \\
\hline Granulocyte colony-stimulating factor receptor (Csf3r) & 8.552 & 0.001 \\
\hline Collagen alpha-1(X) chain (Col10a1) & 2.161 & 0.001 \\
\hline Matrix Gla protein (Mgp) & 1.535 & 0.003 \\
\hline Alpha-fetoprotein (Afp) & 0.637 & 0.001 \\
\hline Lactotransferrin (Ltf) & 0.550 & 0.003 \\
\hline Serum albumin (Alb) & 0.545 & 0.001 \\
\hline Alpha-2-HS-glycoprotein (Ahsg) & 0.364 & 0.001 \\
\hline \multicolumn{3}{|l|}{ Intracellular trafficking, secretion, and vesicular transport } \\
\hline Granulocyte colony-stimulating factor receptor (Csf3r) & 8.552 & 0.001 \\
\hline Sorting nexin-3 (Snx3) & 1.865 & 0.027 \\
\hline Ran-specific GTPase-activating protein (Ranbp1) & 1.641 & 0.001 \\
\hline Mitochondrial fission factor (Mff) & 1.503 & 0.010 \\
\hline Disabled homolog 2 (Dab2) & 1.500 & 0.001 \\
\hline \multicolumn{3}{|l|}{ RNA processing and modification } \\
\hline Prothymosin alpha (Ptma) & 1.890 & 0.016 \\
\hline Serine/arginine-rich splicing factor 5 (Srsf5) & 1.526 & 0.001 \\
\hline \multicolumn{3}{|l|}{ Amino acid transport and metabolism } \\
\hline Chymotrypsinogen B (Ctrb1) & 0.511 & 0.001 \\
\hline Kallikrein 1-related peptidase b1 (Klk1b1) & 0.291 & 0.019 \\
\hline \multicolumn{3}{|l|}{ Inorganic ion transport and metabolism } \\
\hline Lactotransferrin (Ltf) & 0.550 & 0.003 \\
\hline Zinc transporter ZIP8 (Slc39a8) & 0.395 & 0.001 \\
\hline \multicolumn{3}{|l|}{ Carbohydrate transport and metabolism } \\
\hline Glyceraldehyde-3-phosphate dehydrogenase (Gapdh) & 1.547 & 0.001 \\
\hline \multicolumn{3}{|l|}{ Replication, recombination and repair } \\
\hline Prothymosin alpha (Ptma) & 1.890 & 0.016 \\
\hline \multicolumn{3}{|l|}{ Cell cycle control, cell division, chromosome partitioning } \\
\hline Septin-7 (Sept7) & 1.623 & 0.001 \\
\hline \multicolumn{3}{|l|}{ Energy production and conversion } \\
\hline Prothymosin alpha (Ptma) & 1.890 & 0.016 \\
\hline
\end{tabular}

filament that comprise the cell cytoskeleton [23]. Marcksl1 is a member of the Marcks family of proteins that play a pivotal role in actin cytoskeletal organization, the protein kinase C signaling pathway, and the calmodulin signaling pathway [24]. Dsp is a critical component of desmosome structures, which are adhesive junctions at adjacent cell contacts that maintain the structural integrity of tissues that undergo mechanical stress by anchoring the keratin cytoskeleton to the desmosomal plaque [25]. In addition, Sept7, Tmod2, and Sncg are three crucial regulators of cytoskeleton dynamics that are involved in microtubule regulation, keratin network, and actin filament assembly and organization [26-28]. Taken together, these results suggest that GZZSZTW directly targets the cytoskeletal networks of primary chondrocytes. Therefore, GZZSZTW might change cytoskeleton dynamics and enable chondrocytes to fulfill their biological functions, thus providing an effective treatment for OA.

According to the eggNOG annotation, there were 14 differentially expressed proteins that were classified into the category of posttranslational modification, protein turnover, 
TABLE 10: Validation of differentially expressed proteins using PRM assay (GZZSZTW versus Blank).

\begin{tabular}{|c|c|c|c|c|}
\hline Protein name & $\begin{array}{c}\text { Fold change } \\
\text { (iTRAQ) }\end{array}$ & $\begin{array}{c}\mathrm{p} \text { value } \\
\text { (iTRAQ) }\end{array}$ & $\begin{array}{c}\text { Fold change } \\
(\mathrm{PRM})\end{array}$ & $\begin{array}{l}\text { p value } \\
(\mathrm{PRM})\end{array}$ \\
\hline Keratin, type I cytoskeletal 42 (Krt42) & 2.112 & 0.001 & 1.946 & 0.005 \\
\hline Keratin, type II cytoskeletal 5 (Krt5) & 1.973 & 0.001 & 1.283 & 0.022 \\
\hline Keratin, type I cytoskeletal 16 (Krt16) & 2.027 & 0.001 & 1.170 & 0.000 \\
\hline Matrix Gla protein (Mgp) & 1.535 & 0.003 & 3.538 & 0.002 \\
\hline Pregnancy zone protein (Pzp) & 2.088 & 0.001 & 1.733 & 0.000 \\
\hline 60 S ribosomal protein L35 (Rpl35) & 1.537 & 0.001 & 1.923 & 0.021 \\
\hline 40 S ribosomal protein S23 (Rps23) & 1.546 & 0.001 & 1.796 & 0.031 \\
\hline Craniofacial development protein 1 (Cfdp1) & 1.683 & 0.015 & 1.847 & 0.030 \\
\hline Parathymosin (Ptms) & 3.085 & 0.001 & 2.421 & 0.008 \\
\hline Histone H1.5 (Histlh1b) & 2.104 & 0.001 & 1.924 & 0.013 \\
\hline Methyl-CpG-binding protein 2 (Mecp2) & 2.056 & 0.040 & 1.044 & 0.004 \\
\hline Histone H1.1 (Histlhla) & 1.530 & 0.001 & 1.713 & 0.030 \\
\hline
\end{tabular}

and chaperones. The expression levels of 8 proteins were increased, whereas those of 6 proteins were decreased after GZZSZTW treatment. Among the upregulated proteins, Pzp, Ovos, and Cast were proteinase inhibitors that cripple the proteases and prevent the degradation of proteins. Pzp and Ovos are proteinase inhibitors that are able to inhibit all four classes of proteinases [29, 30]. Cast is a specific, suicide inhibitor of calpain, which is a calcium-dependent cysteine protease [31]. These results suggest that GZZSZTW treatment confers the primary chondrocyte the capacity to prevent protein degradation, which is consistent with the effects of GZZSZTW for treating OA. In addition to Marcksll and Sncg, which were involved in cytoskeleton regulation, the expression level of another protein, namely, matrix Gla protein (Mgp), was also significantly increased after GZZSZTW treatment. Mgp is a powerful but developmentally regulated inhibitor of cartilage mineralization. Constitutive Mgp expression in the limb not only inhibits cartilage mineralization but also blocks chondrocyte maturation and intramembranous and endochondral ossification [32]. Therefore, the increased expression level of Mgp upon GZZSZTW treatment plays a key role in the prevention of primary chondrocyte maturation and mineralization, which could be an underlying mechanism for OA treatment with GZZSZTW. The expression levels of several differentially expressed proteins were significantly decreased upon GZZSZTW treatment, including Afp, Ltf, Alb, Ctrb1, Ahsg, and Klk1bl. Among these proteins, Ltf plays a negative role in regulating chondrocyte homeostasis by inhibiting aggrecan synthesis and increasing the levels of catabolic indicators in chondrocytes [33]. Ahsg, also known as fetuin-A, plays a role in inducing the expression of alkaline phosphatase during chondrocyte differentiation [34]. Ctrbl and Klklb are proteinases that perform proteolysis to break down proteins and polypeptides. Ctrbl is the precursor of the proteolytic enzyme chymotrypsin, whereas Klk1b1 is a member of the kallikrein subfamily, which show trypsin- or chymotrypsinlike serine proteases properties $[35,36]$. These results suggest that GZZSZTW treatment significantly decreases the expression of proteinases and prevents the breakdown of the extracellular matrix of chondrocytes. Our results also suggest that GZZSZTW treatment may prevent chondrocytes differentiation.

According to the eggNOG annotation, there were 11 differentially expressed proteins that were classified into the category of signal transduction mechanisms, and the levels of almost all of these proteins were increased subsequent to GZZSZTW treatment. In addition to the proteins that we have previously discussed, including Krt79, Marcksl1, and Ahsg, several other proteins that might play key roles in chondrocytes were identified: Csf3r, Dap, Phlpp2, Sh3glb1, Pth2, Cfdp1, Akap8, and Dab2. Among these proteins, Csf3r is a cell surface receptor for the granulocyte colony-stimulating factor, which plays a pivotal role during the process of cartilage repair [37]. Dap, Phlpp2, and Sh3glb1 are important mediators involved in cell survival and death through regulating multiple signal transduction pathways [38-40]. Pth2, a ligand for the parathyroid hormone 2 receptor, plays a pivotal role in regulating chondrocyte proliferation and differentiation [41]. Akap8 is a member of the A-kinase anchoring protein family that is mainly expressed in the matrix of precartilage but not in fully differentiated cartilage [42]. These results suggest that GZZSZTW regulates chondrocyte survival and death through multiple signal transduction mediators.

According to the eggNOG annotation, there were 11 differentially expressed proteins that were classified into the category of chromatin structure and dynamics, and the levels of almost all of these proteins were increased after GZZSZTW treatment. Among these proteins, Ptms, a small nuclear protein, interacts with the H1 linker histones (e.g., Histlhlb, Histlhld, and Histlhle), which are synthesized during DNA synthesis and are associated with cell proliferation, chromatin remodeling, and gene transcription [43-45]. Mecp2 is a nuclear protein that maintains DNA methylation by regulating multiple regulatory complexes during DNA replication [46]. Hmgal is not only a nuclear protein that is associated with chromatin remodeling but also an important protein for mitochondrial DNA maintenance and organelle function [47]. Gapdh was initially considered as a housekeeping gene. However, Gapdh may be highly upregulated in some cancers 
that are correlated with excessive cell proliferation [48]. Tdrd1 is a direct gene target of the transcription factor Erg that is strongly associated with primary prostate cancer [49]. These results suggest that GZZSZTW treatment increases the expression levels of proteins that regulate chromatin structure and dynamics, which consequently accelerate chondrocyte proliferation.

According to the eggNOG annotation, there were 10 differentially expressed proteins that were classified into the category of translation, ribosomal structure, and biogenesis, and the expression of almost all these proteins was increased after GZZSZTW treatment. The majority of these proteins belonged to the $40 \mathrm{~S}$ and $60 \mathrm{~S}$ ribosomal protein subunits including Rps19, Rps27l, Rpl34, Rpl37, Rps28, Rps23, Rpl35, and Rpl36. These proteins not only participate in translation, ribosomal structure, and biogenesis but also play a crucial role in the regulation of cell proliferation. For instance, an Rps19 deficiency in hematopoietic cells significantly decreased cell proliferation rates, and then Rps19 overexpression in these cells improved or even rescued this event [50]. Overexpression of $\mathrm{Rpl} 34$ promotes cell proliferation and is involved in many types of cancer, including human non-small cell lung cancer, gastric cancer, and pancreatic cancer [51]. Furthermore, RPL36 promotes cell proliferation and G1/S cell cycle progression in glioma [52]. These results suggest that GZZSZTW treatment significantly accelerates the process of translation and aids ribosomal structure and biogenesis by increasing expression levels of the $40 \mathrm{~S}$ and $60 \mathrm{~S}$ ribosomal proteins, and these upregulated proteins, in turn, promote chondrocyte proliferation.

\section{Conclusion}

The present study demonstrates that the Chinese medicinal formulation GZZSZTW, which has been widely used to treat joint diseases, modulates chondrocyte structure, dynamics, and metabolism. Those were achieved by controlling multiple cellular processes, such as DNA replication and transcription, protein synthesis and degradation, cytoskeleton dynamics, and signal transduction in chondrocytes. GZZSZTW treatment significantly increased the levels of cytoskeletal proteins, proteinase inhibitors, signaling regulators, and nuclear proteins. However, GZZSZTW treatment significantly decreased the expression levels of proteinases that are capable of hydrolyzing proteins. Furthermore, GZZSZTW treatment significantly increased the levels of proteins that play key roles in promoting chondrocyte proliferation and prevent chondrocyte differentiation and mineralization. Thus, this study has contributed to the current knowledge of the molecular mechanism by which GZZSZTW acts on chondrocytes. It has also shed light on possible strategies to further prevent and treat cartilage-related diseases by using traditional Chinese medicinal formulations.

\section{Data Availability}

The data used to support the findings of this study are available from the corresponding author upon request.

\section{Conflicts of Interest}

All the authors declare that there are no conflicts of interest.

\section{Authors' Contributions}

Baojin Yao and Bocheng Lu equally contributed to this work.

\section{Acknowledgments}

This work was supported by the TCM Clinical Research Center for Bone Diseases of Jilin Province (Grant no. 20180623048TC), the Science and Technology Development Project of Jilin Province (Grant no. 20170520044JH), the Science and Technology Project of Jilin Provincial Education Department (Grant no. JJKH20170721KJ), and the National Natural Science Foundation of China (Grant no. 81702136).

\section{References}

[1] H. Dang, Q. Wang, H. Wang, M. Yan, and X. Liu, "The integration of chinese material medica into the chinese health care delivery system, an update," Phytotherapy Research, vol. 30, no. 2, pp. 292-297, 2016.

[2] C. Cheng, T. Wu, H. Shang et al., "CONSORT extension for chinese herbal medicine formulas 2017: recommendations, explanation, and elaboration (Traditional Chinese Version)," Annals of Internal Medicine, vol. 167, no. 2, 2017.

[3] L. Zhang, J. Hu, and K. A. Athanasiou, "The role of tissue engineering in articular cartilage repair and regeneration," Critical Reviews in Biomedical Engineering, vol. 37, no. 1-2, pp. $1-57,2009$.

[4] M. Z. Ruan, V. Cerullo, R. Cela et al., "Treatment of osteoarthritis using a helper-dependent adenoviral vector retargeted to chondrocytes," Molecular Therapy - Methods \& Clinical Development, vol. 3, p. 16008, 2016.

[5] B. Yao, B. Lu, H. Gao, M. Zhang, X. Leng, and D. Zhao, "Guzhi zengsheng zhitongwan, a traditional chinese medicinal formulation, stimulates chondrocyte proliferation through control of multiple genes involved in chondrocyte proliferation and differentiation," Evidence-Based Complementary and Alternative Medicine, vol. 2018, Article ID 7265939, 10 pages, 2018.

[6] M. Gosset, F. Berenbaum, S. Thirion, and C. Jacques, "Primary culture and phenotyping of murine chondrocytes," Nature Protocols, vol. 3, no. 8, pp. 1253-1260, 2008.

[7] X. Bi, Y. Wang, X. Hu, and X. Liao, "iTRAQ-based proteomic analysis of sublethally injured escherichia coli O157:H7 cells induced by high pressure carbon dioxide," Frontiers in Microbiology, vol. 8, Article ID 2544, 2017.

[8] T. Suriyanarayanan, L. Qingsong, L. T. Kwang, L. Y. Mun, T. Truong, and C. J. Seneviratne, "Seneviratne, Quantitative proteomics of strong and weak biofilm formers of enterococcus faecalis reveals novel regulators of biofilm formation," Molecular and Cellular Proteomics, vol. 17, no. 4, pp. 643-654, 2018.

[9] J. Zhang, P. Huang, C. Wu et al., "Preliminary observation about alteration of proteins and their potential functions in spinal cord of SOD1 G93A transgenic mice," International Journal of Biological Sciences, vol. 14, no. 10, pp. 1306-1320, 2018.

[10] J. Wang, H. Mei, C. Zheng et al., “The metabolic regulation of sporulation and parasporal crystal formation in Bacillus 
thuringiensis revealed by transcriptomics and proteomics," Molecular \& Cellular Proteomics, vol. 12, no. 5, pp. 1363-1376, 2013.

[11] J. Tang, Y. Yu, H. Zheng et al., "ITRAQ-based quantitative proteomic analysis of Cynops orientalis limb regeneration," BMC Genomics, vol. 18, article 750, 2017.

[12] B. Wen, C. Du, G. Li et al., "IPeak: An open source tool to combine results from multiple MS/MS search engines," Proteomics, vol. 15, no. 17, pp. 2916-2920, 2015.

[13] B. Wen, R. Zhou, Q. Feng, Q. Wang, J. Wang, and S. Liu, "IQuant: An automated pipeline for quantitative proteomics based upon isobaric tags," Proteomics, vol. 14, no. 20, pp. $2280-$ 2285, 2014.

[14] J. Xu, L. Zhang, J. Hou et al., "iTRAQ-based quantitative proteomic analysis of the global response to $17 \beta$-estradiol in estrogen-degradation strain Pseudomonas putida SJTE-1," Scientific Reports, vol. 7, no. 1, Article ID 41682, 2017.

[15] J. Chen, H. Xiao, Y. Gui et al., "Characterization of the verticillium dahliae exoproteome involves in pathogenicity from cotton-containing medium," Frontiers in Microbiology, vol. 7, 2016.

[16] Y. R. Musa, S. Boller, M. Puchalska, R. Grosschedl, and G. Mittler, "Comprehensive proteomic investigation of ebf1 heterozygosity in Pro-B lymphocytes utilizing data independent acquisition," Journal of Proteome Research, vol. 17, no. 1, pp. 7685, 2018.

[17] C. K. O’Neil, J. T. Hanlon, and Z. A. Marcum, “Adverse effects of analgesics commonly used by older adults with osteoarthritis: focus on non-opioid and opioid analgesics," American Journal of Geriatric Pharmacotherapy, vol. 10, no. 6, pp. 331-342, 2012.

[18] B. Chen, H. Zhan, J. Marszalek et al., "Traditional chinese medications for knee osteoarthritis pain: a meta-analysis of randomized controlled trials," American Journal of Chinese Medicine, vol. 44, no. 4, pp. 677-703, 2016.

[19] A. J. Sophia Fox, A. Bedi, and S. A. Rodeo, "The basic science of articular cartilage: structure, composition, and function," Sports Health, vol. 1, no. 6, pp. 461-468, 2009.

[20] J. Huerta-Cepas, D. Szklarczyk, K. Forslund et al., "EGGNOG 4.5: A hierarchical orthology framework with improved functional annotations for eukaryotic, prokaryotic and viral sequences," Nucleic Acids Research, vol. 44, no. 1, pp. D286D293, 2016.

[21] E. J. Blain, "Involvement of the cytoskeletal elements in articular cartilage homeostasis and pathology," International Journal of Clinical and Experimental Pathology, vol. 90, no. 1, pp. 1-15, 2009.

[22] J. Schweizer, P. E. Bowden, P. A. Coulombe et al., "New consensus nomenclature for mammalian keratins," The Journal of Cell Biology, vol. 174, no. 2, pp. 169-174, 2006.

[23] H. U. Stotz and S. R. Long, "Expression of the pea (Pisum sativum L.) $\alpha$-tubulin gene TubA1 is correlated with cell division activity," Plant Molecular Biology, vol. 41, no. 5, pp. 601-614, 1999.

[24] A. E. Finlayson and K. W. Freeman, "A cell motility screen reveals role for MARCKS-related protein in adherens junction formation and tumorigenesis," PLoS ONE, vol. 4, no. 11, article e7833, 2009.

[25] L. V. Albrecht, L. Zhang, J. Shabanowitz et al., "GSK3and PRMT-1-dependent modifications of desmoplakin control desmoplakin-cytoskeleton dynamics," The Journal of Cell Biology, vol. 208, no. 5, pp. 597-612, 2015.
[26] J. N. Rao, Y. Madasu, and R. Dominguez, "Mechanism of actin filament pointed-end capping by tropomodulin," Science, vol. 345, no. 6195, pp. 463-467, 2014.

[27] T. Tada, A. Simonetta, M. Batterton, M. Kinoshita, D. Edbauer, and M. Sheng, "Role of Septin cytoskeleton in spine morphogenesis and dendrite development in neurons," Current Biology, vol. 17, no. 20, pp. 1752-1758, 2007.

[28] H. Zhang, A. Kouadio, D. Cartledge, and A. K. Godwin, "Role of gamma-synuclein in microtubule regulation," Experimental Cell Research, vol. 317, no. 10, pp. 1330-1339, 2011.

[29] P. Marie, V. Labas, A. Brionne et al., "Data set for the proteomic inventory and quantitative analysis of chicken eggshell matrix proteins during the primary events of eggshell mineralization and the active growth phase of calcification," Data in Brief, vol. 4, pp. 430-436, 2015.

[30] D. A. T. Nijholt, L. Ijsselstijn, M. M. Van Der Weiden et al., "Pregnancy zone protein is increased in the Alzheimer's disease brain and associates with senile plaques," Journal of Alzheimer's Disease, vol. 46, no. 1, pp. 227-238, 2015.

[31] M. V. Rao, M. K. McBrayer, J. Campbell et al., "Specific calpain inhibition by calpastatin prevents tauopathy and neurodegeneration and restores normal lifespan in tau P301L Mice," The Journal of Neuroscience, vol. 34, no. 28, pp. 9222-9234, 2014.

[32] K. Yagami, J.-Y. Suh, M. Enomoto-Iwamoto et al., "Matrix GLA protein is a developmental regulator of chondrocyte mineralization and, when constitutively expressed, blocks endochondral and intramembranous ossification in the limb," The Journal of Cell Biology, vol. 147, no. 5, pp. 1097-1108, 1999.

[33] N. Brandl, A. Zemann, I. Kaupe et al., "Signal transduction and metabolism in chondrocytes is modulated by lactoferrin," Osteoarthritis and Cartilage, vol. 18, no. 1, pp. 117-125, 2010.

[34] Y. Ishikawa, L. N. Y. Wu, W. B. Valhmu, and R. E. Wuthier, "Fetuin and alpha-2HS glycoprotein induce alkaline phosphatase in epiphyseal growth plate chondrocytes," Journal of Cellular Physiology, vol. 149, no. 2, pp. 222-234, 1991.

[35] S. Curvers, P. Brixius, T. Klauser et al., "Human chymotrypsinogen $\mathrm{B}$ production with Pichia pastoris by integrated development of fermentation and downstream processing. Part 1. Fermentation," Biotechnology Progress, vol. 17, no. 3, pp. 495502, 2001.

[36] G. Sotiropoulou, G. Pampalakis, and E. P. Diamandis, "Functional roles of human Kallikrein-related peptidases," The Journal of Biological Chemistry, vol. 284, no. 48, pp. 32989-32994, 2009.

[37] A. Marmotti, D. E. Bonasia, M. Bruzzone et al., "Human cartilage fragments in a composite scaffold for single-stage cartilage repair: an in vitro study of the chondrocyte migration and the influence of TGF- $\beta 1$ and G-CSF," Knee Surgery, Sports Traumatology, Arthroscopy, vol. 21, no. 8, pp. 1819-1833, 2013.

[38] L. P. Deiss, E. Feinstein, H. Berissi, O. Cohen, and A. Kimchi, "Identification of a novel serine/threonine kinase and a novel 15$\mathrm{kD}$ protein as potential mediators of the $\gamma$ interferon-induced cell death," Genes \& Development, vol. 9, no. 1, pp. 15-30, 1995.

[39] F. Rizvi, S. Shukla, and P. Kakkar, "Essential role of $\mathrm{PH}$ domain and leucine-rich repeat protein phosphatase 2 in Nrf2 suppression via modulation of Akt/GSK3b/Fyn kinase axis during oxidative hepatocellular toxicity," Cell Death \& Disease, vol. 5, no. 3, Article ID el153, 2014.

[40] Y. Takahashi, C. L. Meyerkord, and H. G. Wang, "Bif1/Endophilin B1: A candidate for crescent driving force in autophagy," Cell Death \& Differentiation, vol. 16, no. 7, pp. 947955, 2009. 
[41] D. K. Panda, D. Goltzman, and A. C. Karaplis, "Defective postnatal endochondral bone development by chondrocytespecific targeted expression of parathyroid hormone type 2 receptor," American Journal of Physiology-Endocrinology and Metabolism, vol. 303, no. 12, pp. E1489-E1501, 2012.

[42] Q. Zhang, D. W. Carr, K. M. Lerea, J. D. Scott, and S. A. Newman, "Nuclear localization of type II cAMP-dependent protein kinase during limb cartilage differentiation is associated with a novel developmentally regulated A-kinase anchoring protein," Developmental Biology, vol. 176, no. 1, pp. 51-61, 1996.

[43] S. P. Hergeth and R. Schneider, "The H1 linker histones: Multifunctional proteins beyond the nucleosomal core particle," EMBO Reports, vol. 16, no. 11, pp. 1439-1453, 2015.

[44] G. Martic, Z. Karetsou, K. Kefala et al., "Parathymosin affects the binding of linker histone $\mathrm{H} 1$ to nucleosomes and remodels chromatin structure," The Journal of Biological Chemistry, vol. 280, no. 16, pp. 16143-16150, 2005.

[45] M. Okuwaki, M. Abe, M. Hisaoka, and K. Nagata, "Regulation of cellular dynamics and chromosomal binding site preference of linker histones H1.0 and H1.X," Molecular and Cellular Biology, vol. 36, no. 21, pp. 2681-2696, 2016.

[46] H. Kimura and K. Shiota, "Methyl-CpG-binding protein, $\mathrm{MeCP} 2$, is a target molecule for maintenance DNA methyltransferase, Dnmt1," The Journal of Biological Chemistry, vol. 278, no. 7, pp. 4806-4812, 2003.

[47] G. A. Dement, S. C. Maloney, and R. Reeves, "Nuclear HMGA1 nonhistone chromatin proteins directly influence mitochondrial transcription, maintenance, and function," Experimental Cell Research, vol. 313, no. 1, pp. 77-87, 2007.

[48] D. Wang, D. R. Moothart, D. R. Lowy, and X. Qian, “The expression of glyceraldehyde-3-phosphate dehydrogenase associated cell cycle (GACC) genes correlates with cancer stage and poor survival in patients with solid tumors," PLoS ONE, vol. 8, no. 4, article e61262, 2013.

[49] J. L. Boormans, H. Korsten, A. J. C. Ziel-Van Der Made et al., "Identification of TDRD1 as a direct target gene of ERG in primary prostate cancer," International Journal of Cancer, vol. 133, no. 2, pp. 335-345, 2013.

[50] I. Hamaguchi, J. Flygare, H. Nishiura et al., "Proliferation deficiency of multipotent hematopoietic progenitors in ribosomal protein S19 (RPS19) - Deficient Diamond-Blackfan anemia improves following RPS19 gene transfer," Molecular Therapy, vol. 7, no. 5 I, pp. 613-622, 2003.

[51] F. Wei, L. Ding, Z. Wei et al., "Ribosomal protein L34 promotes the proliferation, invasion and metastasis of pancreatic cancer cells," Oncotarget, vol. 7, no. 51, pp. 85259-85272, 2016.

[52] Y.-W. Hu, C.-M. Kang, J.-J. Zhao et al., "LncRNA PLAC2 downregulates RPL36 expression and blocks cell cycle progression in glioma through a mechanism involving STAT1," Journal of Cellular and Molecular Medicine, vol. 22, no. 1, pp. 497-510, 2018. 

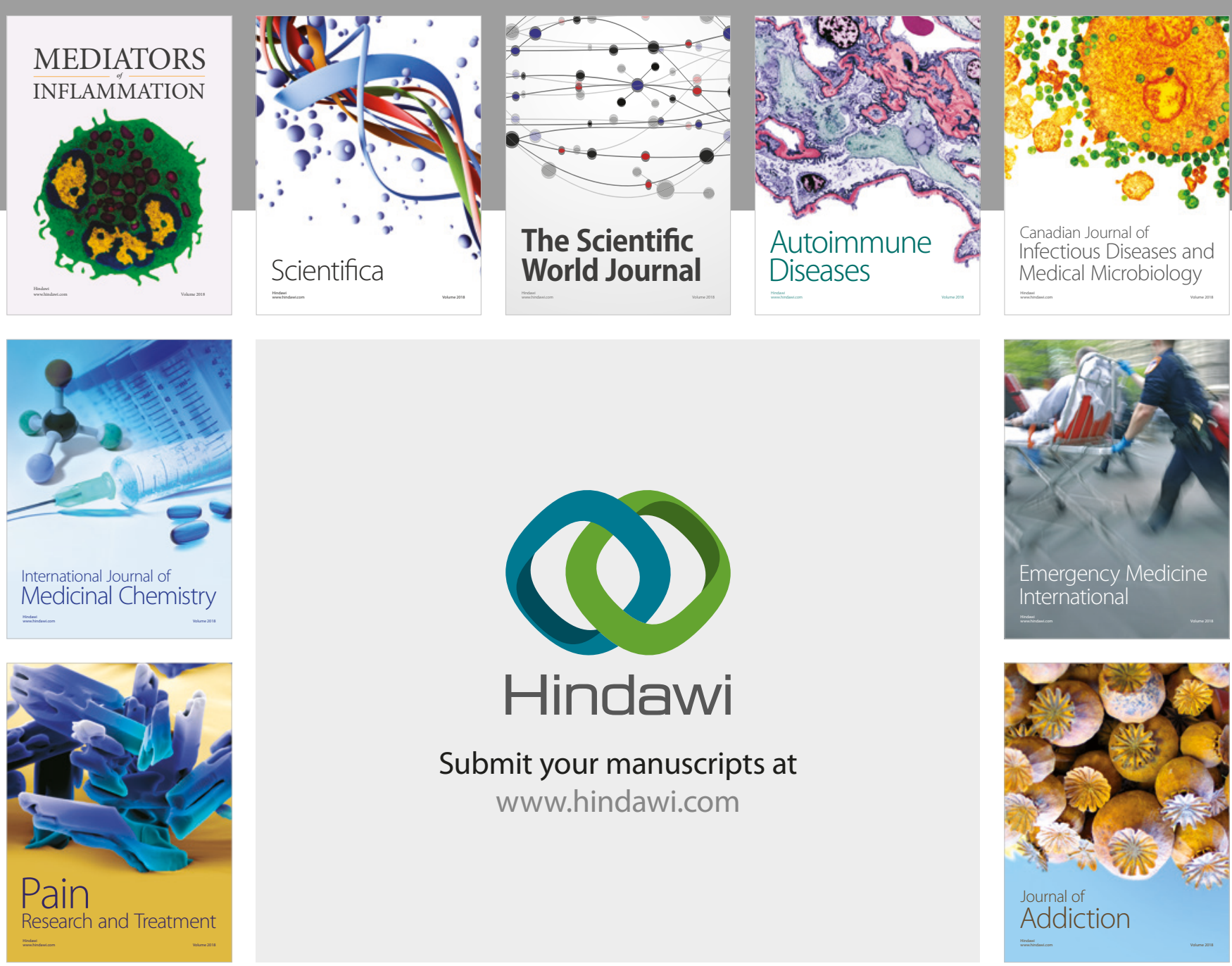

Canadian Journal of
Infectious Diseases and Medical Microbiology

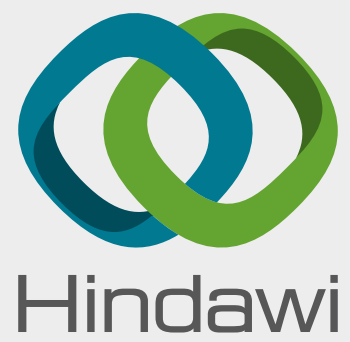

Submit your manuscripts at

www.hindawi.com
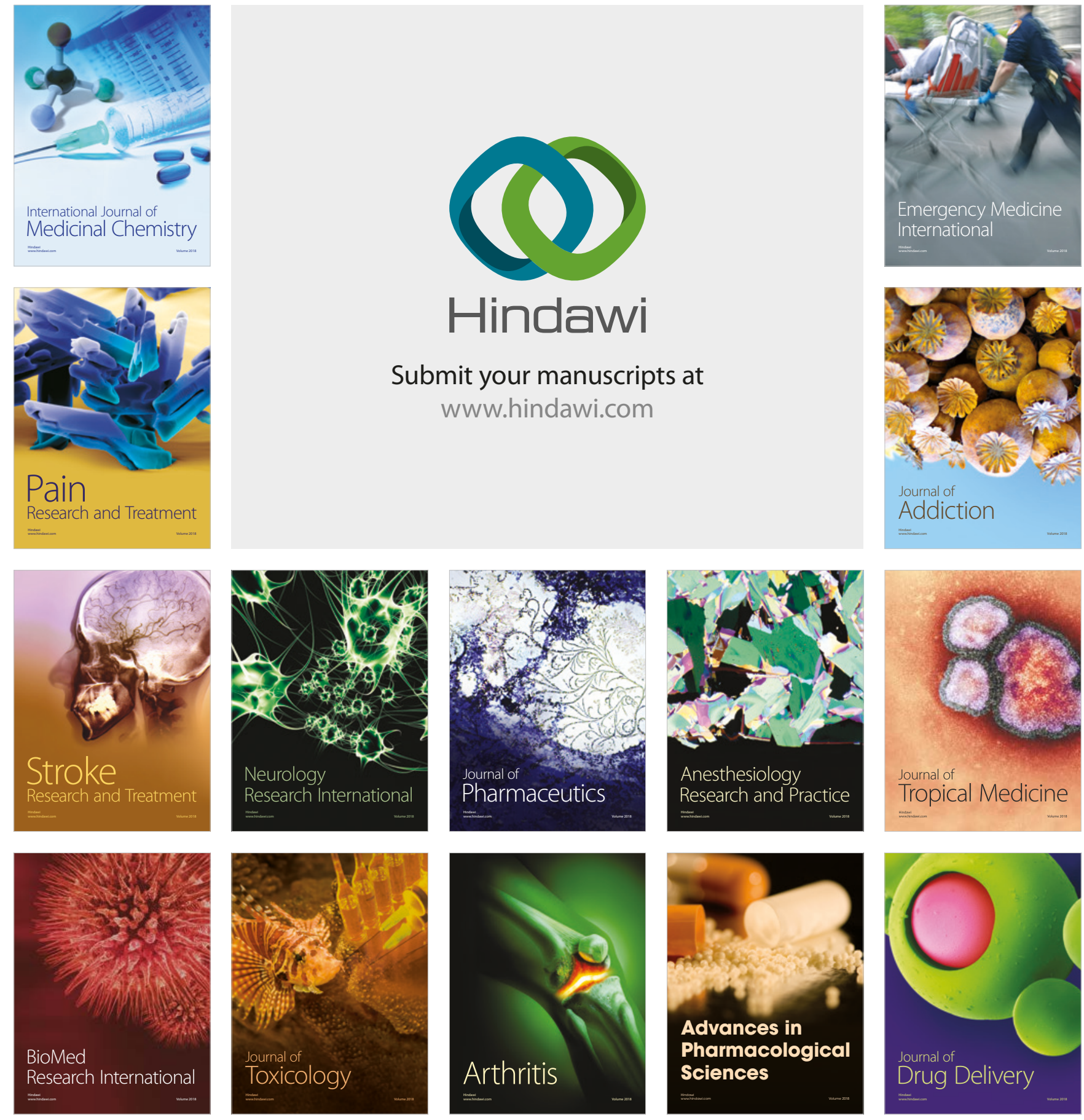